\title{
A family of difference sets having \\ -1 as an invariant
}

\author{
By Masahiko Miчамото
}

(Received May 29, 1982)

A construction is given for difference sets having -1 as an invariant, whose parameters are

$$
v=\frac{1}{2} 3^{s+1}\left(3^{s+1}-1\right), \quad k=\frac{1}{2} 3^{s}\left(3^{s+1}+1\right), \lambda=\frac{1}{2} 3^{s}\left(3^{s}+1\right), \quad n=3^{2 s}(s \text { even }) .
$$

Let $G$ be a finite group of order $v$. A subset $D$ of order $k$ is called a difference set in $G$ with parameters $(v, k, \lambda, n)$ in case every non-identity element $g$ in $G$ can be expressed in exactly $\lambda$ way as $g=d_{1}^{-1} d_{2}$ with $d_{1}, d_{2} \in D$. The parameter $n$ is defined by $n=k-\lambda$. For any integer $t$, let $D(t)$ denote the image of $D$ under the mapping $g \rightarrow g^{t}, g \in G$. If the mapping is an automorphism of $G$ and $D(t)$ is a translate of $D$, then $t$ is called a multiplier of $D$. But even if it is not an automorphism, $t=-1$ has an important property, that is, it makes a non-direct graph which has a regular automorphism.

In this paper, we will show an infinite series of difference sets having -1 as an invariant. Spence [1] showed a family of difference set with parameters

$$
v=\frac{1}{2} 3^{s+1}\left(3^{s+1}-1\right), k=\frac{1}{2} 3^{s}\left(3^{s+1}+1\right), \lambda=\frac{1}{2} 3^{s}\left(3^{s}+1\right), n=3^{2 s} .
$$

By modification of his argument, we will prove the following theorem.

THEOREM. There exists a difference set with parameter

$$
v=\frac{1}{2} 3^{s+1}\left(3^{s+1}-1\right), \quad k=\frac{1}{2} 3^{s}\left(3^{s+1}+1\right), \lambda=\frac{1}{2} 3^{s}\left(3^{s}+1\right), \quad n=3^{2 s}
$$

which has -1 as an invariant for each even integer $s \geq 2$.

Proof. Let $E$ denote the additive group of $G F\left(3^{s+1}\right)$ and $K_{1}$ be the multiplicative group of $G F\left(3^{s+1}\right)$, where $s$ is an even integer $\geq 2$. Then since $s$ is even, we have $K_{1}=Z / 2 Z \times K$ for a subgroup $K$ of odd order. Set $G=E^{*} K$ be the semi-direct product of $E$ by $K$. Then we have the following ; 
a) $|G|=\frac{1}{2} 3^{s+1}\left(3^{s+1}-1\right)$,

b) $K$ is a cyclic subgroup of order $r=\frac{1}{2}\left(3^{s+1}-1\right)$,

c) $K$ acts on $E^{\sharp}$ as fixed point free automorphisms, and

d) $K$ permutes all hyperplanes of $E$ transitively and no elements of $K^{\sharp}$ fix a hyperplane of $\mathrm{E}$.

Let $H$ be a hyperplane of $E$ and $k_{1}=1, k_{2}, \cdots, k_{r}$ be the elements of $K$. Then we will show that

$$
D=(E-H) * k_{1} \cup \bigcup_{i=1}^{r}\left(H^{\sqrt{k_{i}}}{ }^{-1}\right) * k_{i}
$$

is a difference set in $G$ having -1 as an invariant, where $\sqrt{k}$ is a square of $k$ in $K$, which is well defined since $K$ has an odd order. Using the group ring notation for $Z E$, it is readily seen that (cf. [1])

$$
\begin{aligned}
& H^{\sqrt{\bar{k}_{1}}}+H^{-1}{\overline{\bar{k}_{2}}}^{-1}+\cdots+H^{\sqrt{k_{r}}}=3^{s} . \quad 1_{E}+\frac{1}{2}\left(3^{s}-1\right) E, \\
& H^{\sqrt{\bar{k}_{i}^{-1}}} H^{\sqrt{k_{i}}}{ }^{-1}=3^{s} H^{\sqrt{k_{i}}}{ }^{-1}, \quad H^{\sqrt{k_{i}}}{ }^{-1} H^{\bar{k}_{j}}=3^{s-1} E(i \neq j), \\
& (E-H)(E-H)=3^{s}(H+E), \quad \text { and }(E-H) H^{\sqrt{k_{i}}}{ }^{-1}=2 \cdot 3^{s-1} E(i \neq 1) .
\end{aligned}
$$

To verify that $D$ is a difference set in $G$ it is sufficient to show that $D(-1) D=n 1_{G}+\lambda G$, where $n, \lambda$ are as above. We can easily check $D(-1)$ $=D$. Using the above results, we obtain

$$
\begin{aligned}
& D(-1) D=(E-H)(E-H) * k_{1}+\sum_{j=2}^{r}\left(H^{\sqrt{k_{i}} * k_{i}}{ }^{-1}\right)\left(H^{\sqrt{k_{i}}}{ }^{-1} * k_{i}\right) \\
& +\sum_{2 \leq i \neq j \leq r}\left(H^{\sqrt{k_{i}} * k_{i}}{ }^{-1}\right)\left(H^{\sqrt{k_{j}}{ }^{-1}} * k_{j}\right) \\
& +(E-H) \sum_{j=2}^{r} H^{\sqrt{k_{j}}-{ }^{-1}} * k_{j}+\left(\sum_{i=2}^{r} H^{\sqrt{k_{i}} * k_{i}}{ }^{-1}\right)(E-H) * 1_{K} \\
& =3^{2}(E+H) * 1_{K}+\sum_{i=2}^{r} H^{\overline{k_{i}}} H^{\overline{k_{i}}} * 1_{K}+\sum_{2 \leq i \neq j \leq r}^{i} H^{\overline{k_{i}}} H^{\sqrt{k_{j}}{ }^{-1} k_{i} * k_{i}{ }^{-1} k_{j}} \\
& +2 \cdot 3^{s-1} E^{*}\left(K-1_{K}\right)+\sum_{i=2}^{r} H^{\overline{k_{i}}}(E-H)^{\sqrt{k_{i}} * k_{i}{ }^{-1}} \\
& =3^{2}(E+H) * 1_{K}+\sum_{i=2}^{r} 3^{s} H^{\sqrt{k_{i}} *} 1_{K}+\sum_{2 \leq i \neq j \leq r} 3^{s-1} E^{*} k_{i}{ }^{-1} k_{j} \\
& +2 \cdot 3^{s-1} E^{*}\left(K-1_{K}\right)+2 \cdot 3^{s-1} E^{*}\left(K-1_{K}\right) \\
& =3^{s} E^{*} 1_{K}+3^{s}\left(3^{s} \cdot 1_{E}+\frac{1}{2}\left(3^{s}-1\right) E\right) * 1_{K}+3^{s-1}(r+2) E^{*}\left(K-1_{K}\right) \\
& =3^{2 s} \cdot 1_{G}+\frac{1}{2} 3^{s}\left(3_{s}+1\right) E^{*} 1_{K}+3^{s-1}\left(\frac{1}{2}\left(3^{s+1}-1\right)+2\right) E^{*}\left(K-1_{K}\right) \\
& =3^{2 s} \cdot 1_{G}+\frac{1}{2} 3^{s}\left(3^{s}+1\right) G \text {. }
\end{aligned}
$$


So we have proved that $D$ is a difference set having -1 as an invariant. This completes the proof of Theorem.

\section{Reference}

[1] E. SPENCE: A family of difference sets, J. Combin. Theory ser. A 22 (1977), 103-106.

Department of Mathematics

Ehime University 